\title{
Synthesis and Gas Permeation Properties of Star-like Poly(ethylene oxide)s Using Hyperbranched Polyimide as Central Core
}

\author{
Yan Yin, Liming Yang, Makoto Yoshino, Jianhua FAng, Kazuhiro TANaKA, \\ Hidetoshi KiтA, and Ken-ichi Окамото ${ }^{\dagger}$ \\ Department of Advanced Materials Science \& Engineering, Faculty of Engineering, Yamaguchi University, \\ 2-16-1 Tokiwadai, Ube 755-8611, Japan
}

(Received September 22, 2003; Accepted January 26, 2004)

\begin{abstract}
A series of star-like poly(ethylene oxide)s were synthesized using anhydride-terminated hyperbranched polyimides as the central cores and poly(ethylene oxide)s (PEOs) as the linear arms. Their physical and gas permeation properties were investigated in comparison with those of PEO segmented block copolyimides (PEOPIs). The solubility of star-like PEOs was affected by the terminal groups. The amine-terminated star-like PEOs displayed better solubility property than the methoxy- and acetamido-terminated ones. The DSC and dynamic mechanical spectroscopy suggested the morphology of star-like PEOs was different from that of PEO-PIs. The thermo-mechanical property of amine-terminated star-like PEOs was significantly improved by cross-linking with ethylene glycol diglycidyl ether. The star-like PEO membranes were much more permeable to $\mathrm{CO}_{2}$ than to $\mathrm{N}_{2}$ and even to $\mathrm{H}_{2}$. However, their $\mathrm{CO}_{2}$ separation performance was slightly lower than that of PEO-PIs, probably due to the difference in the morphology. The $\mathrm{AgBF}_{4}$-doped star-like PEO membrane with a loading of $40 \mathrm{wt} \%$ (corresponding to $67 \mathrm{wt} \%$ in PEO matrix) showed a very high ideal selectivity of $\mathrm{C}_{2} \mathrm{H}_{4} / \mathrm{C}_{2} \mathrm{H}_{6}$ of more than 100 with a low ethylene permeability of $1.5 \times$ $10^{-10} \mathrm{~cm}^{3}$ (STP) $\mathrm{cm}^{-1} \mathrm{~s}^{-1} \mathrm{~cm} \mathrm{Hg}^{-1}$ in single-component permeation at $2 \mathrm{~atm}$ and $308 \mathrm{~K}$. However, the permselectivity decreased down to 14 in mixed gas permeation. The permeation behavior was discussed based on the solubility and diffusivity.

KEY WORDS Star-like Polymer / Polyethylene Oxide / Membrane Separation / Polymer Electrolyte Membrane / Carbon Dioxide Separation / Ethylene/Ethane Separation /
\end{abstract}

Membrane-based gas separations have attracted much attention in the past decade because they offer significant advantages over traditional separation processes such as low energy consumption, low capital investment cost and simple operation. A large number of polymeric materials have been studied for this application. However, most of the materials developed so far are limited mainly to linear type polymers. For example, many studies have been devoted to the investigation of the relationship between chemical structure and gas permeation property of polyimides (PIs) in order to develop membranes with both high permeability and high selectivity. ${ }^{1-5}$ On the other hand, very few attempts have been made using nonlinear (multibranched or multiarmed) polymers as membrane materials for gas separations. Hyperbranched PIs can be simply prepared by direct "one-step" polymerization of tri-functional amines with common acid dianhydrides. ${ }^{6}$ Hyperbranched PIs have rather poor membrane-forming ability, but their tough membranes can be fabricated by crosslinking treatment during membrane casting using the terminal functional groups. ${ }^{7}$ Their gas permeation properties significantly depend on both the terminal functional groups and the crosslinking. ${ }^{7}$

Poly(ethylene oxide) (PEO) and PEO-containing polymers have been extensively studied for gas separations. ${ }^{8-15}$ Poly(ethylene-oxide imide) segmented block copolymers (PEO-PIs) have microphase-separated structures consisting of rubbery PEO domains and glassy PI domains and have excellent separation performance of polar/nonpolar gas pairs such as $\mathrm{CO}_{2} / \mathrm{N}_{2}$ and $\mathrm{CO}_{2} / \mathrm{H}_{2}$. The high separation performance is due to the high solubility of $\mathrm{CO}_{2}$ in PEO domains, and PI domains contribute to the mechanical properties and film-forming ability. ${ }^{8}$ Therefore, the separation performance is significantly affected by the morphology of the microphase-separated structure, which is also affected by hard-segment polymer structures. ${ }^{9}$ Furthermore, PEO and a poly(tetramethylene-oxide amide) segmented block copolymer (PTMO-PA12) have been used as materials for solid polymer electrolyte membranes to separate olefin/paraffin mixtures, because polyethers form polymer electrolyte complexes with a number of salts, including silver salts. ${ }^{16,17}$

If the microphase-separated structure is favorable for gas separation, the multi-armed star-like polymers

${ }^{\dagger}$ To whom correspondence should be addressed (Tel: 0836-85-9660, Fax: 0836-85-9601, E-mail: okamotok@ yamaguchi-u.ac.jp). 


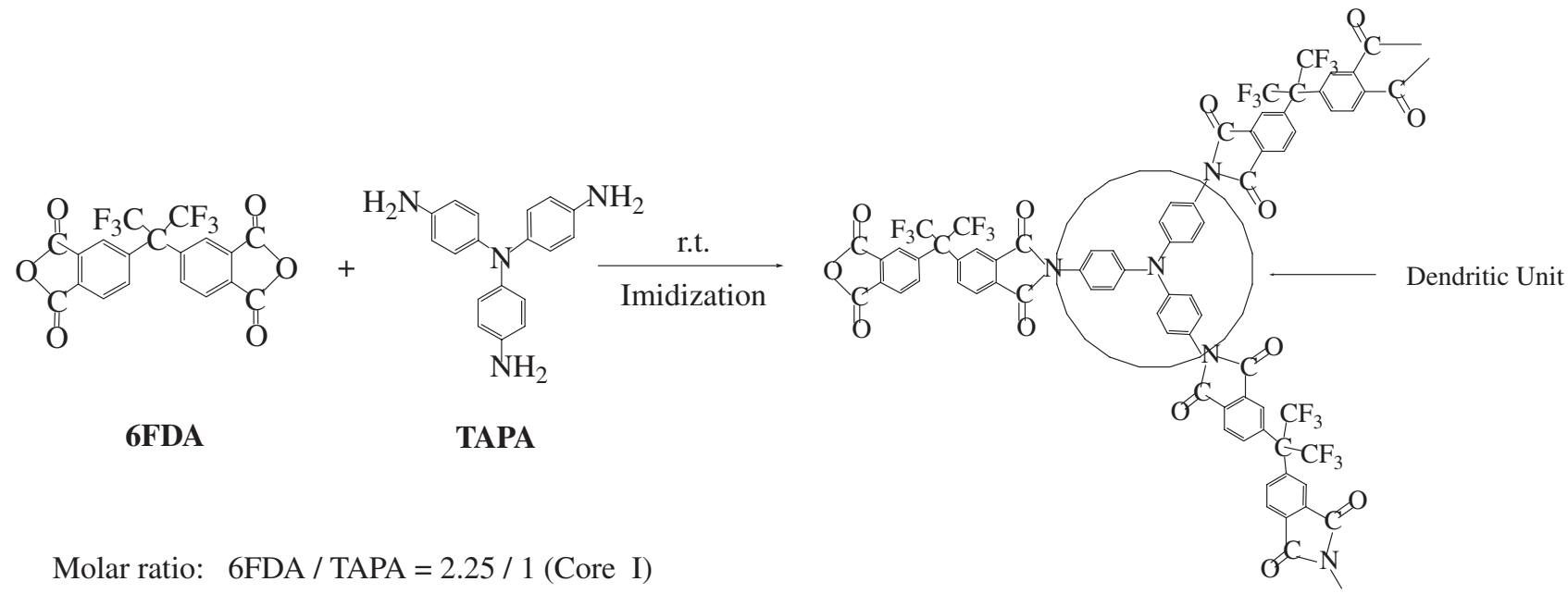

$2.07 / 1$ (Core II)

Core (Anhydride-terminated

$2.00 / 1$ (Core III)

hyperbranched polyimide)

Figure 1. Schematic diagram of synthesis of anhydride-terminated hyperbranched polyimide (Core).

may also be suitable for gas separation applications. Multi-armed star-like polymers are three-dimensional macromolecules, in which a large number of linear arms of similar molecular weight emanate from a central core. Although many star-like polymers have been widely studied, ${ }^{18}$ there is no report on star-like polymers used for gas separation. The three-dimensional microphase-separated structure in star-like polymers may be quite different from that of linear segmented ones, and the intermolecular packing of star-like polymers may also be different from the intersegmental packing of linear polymer chains. Thus, star-like polymers might exhibit different gas permeation properties from linear ones and are expected to be a novel class of membrane materials for gas separations.

In this study, we report on synthesis of a series of star-like polymers consisting of hyperbranched PIs as cores and PEOs as arms and their physical and gas permeation properties.

\section{EXPERIMENTAL}

\section{Materials}

Tris(4-aminophenyl)amine (TAPA) was synthesized by reducing tris(4-nitrophenyl)amine with hydrazine monohydrate in the presence of $\mathrm{Pd} / \mathrm{C}$ $(10 \mathrm{wt} \%){ }^{6} \quad$ Poly(ethylene glycol)methyl ether (PEGME) with an average block length, $n$, of poly(ethylene oxide) (PEO) of 113 (PEO1) and 45 (PEO2), and bis(3-aminopropyl)-polyethylene oxide (BAPEO) with $n$ of 62 (PEO3) were purchased from Aldrich Chemical Co., Inc. and used as received. 2,2-Bis(3,4-dicarboxyphenyl) hexafluoropropane dianhydride (6FDA) was purified by sublimation prior to use. $N, N$-dimethylacetamide (DMAc) was distilled under reduced pressure and dehydrated with $4 \AA$ molecular sieves. Other materials were used as received.

\section{Polymerization}

Anhydride-terminated 6FDA-TAPA Hyperbranched Polyimide (Core). Anhydride-terminated 6FDATAPA hyperbranched PIs were prepared according to our previously reported method. ${ }^{6}$ The molar ratio of 6FDA/TAPA was controlled to be $2: 1,2.07: 1$ and 2.25:1 to give three kinds of anhydride terminated hyperbranched PIs, which were used as central cores. The schematic diagram of the synthesis of the cores is shown in Figure 1. To obtain core I, the synthesis procedure is as follows. $1.5 \mathrm{~g}(3.38 \mathrm{mmol})$ of 6FDA was dissolved in $30 \mathrm{~mL}$ of DMAc in a $200 \mathrm{~mL}$ thoroughly dried four-neck flask under nitrogen flow. To this solution was added dropwise $0.435 \mathrm{~g}(1.50 \mathrm{mmol})$ of TAPA in $15 \mathrm{~mL}$ of DMAc through a syringe over a period of $6 \mathrm{~h}$. The reaction mixture was further stirred for $3 \mathrm{~h}$. Then a mixture solution of $3.0 \mathrm{~g}$ of triethylamine and $9.0 \mathrm{~g}$ of acetic anhydride was added, and the reaction mixture was stirred at room temperature for $20 \mathrm{~h}$. The resulting mixture was poured into diethyl ether, the precipitate was dried in vacuo. The elementary analysis for core I (6FDA/TAPA $=2.25: 1$, $\mathrm{C}_{243} \mathrm{H}_{102} \mathrm{~N}_{16} \mathrm{O}_{42} \mathrm{~F}_{54}$ ): Calcd: C, 59.02; H, 2.06; N, 4.53. Found: 58.49; H, 2.04; N, 4.56. For core II (6FDA/TAPA $=2.07: 1$ ): Calcd: C, 59.54; H, 2.11; $\mathrm{N}, 4.83$. Found: $59.55 ; \mathrm{H}, 2.13 ; \mathrm{N}, 4.37$.

Methoxy-terminated Star-like PEO. The schematic diagram of synthesis of star-like PEOs (abbreviated to SPEOs here after) with different arms is shown in Figure 2. To a $100 \mathrm{~mL}$ thoroughly dried four-neck flask equipped with a Dean-Stark trap were charged $1.0 \mathrm{~g}$ of hyperbranched PI (Core I: 6FDA/TAPA = 


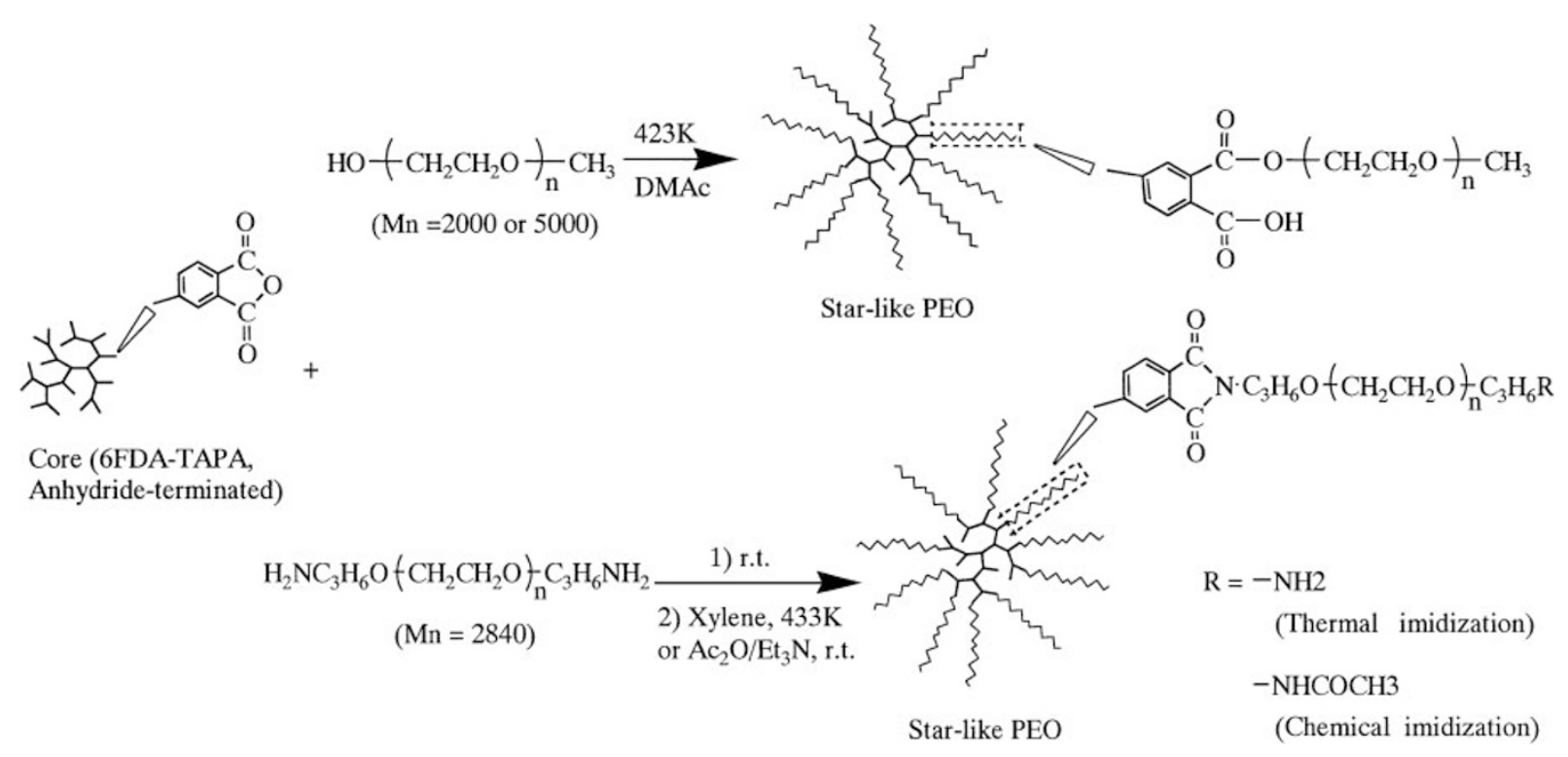

Figure 2. Schematic diagram of synthesis of star-like PEO.

2.25:1, Anhydride: $1.16 \mathrm{mmol}$ ) in $20 \mathrm{~mL}$ of DMAc, together with $12 \mathrm{~mL}$ of $m$-xylene, with stirring under nitrogen flow. The solution was heated to $433 \mathrm{~K}$ for $30 \mathrm{~min}$ and then cooled to room temperature. To this solution, $7.0 \mathrm{~g} \quad(1.4 \mathrm{mmol})$ of PEGME (PEO1, $\left.M_{\mathrm{n}}=5000\right)$ was added and the mixture was heated to $403-423 \mathrm{~K}$ for $48 \mathrm{~h}$. The resulting solution was poured into hexane $(\mathrm{Hx})$ and the precipitate was dried in vacuo. The crude product was purified by extracting the unreacted PEGME with a mixture of benzene $(\mathrm{Bz})$ and $\mathrm{Hx}$ (volume ratio of $\mathrm{Bz} / \mathrm{Hx}=2.5: 1$ ). The $\mathrm{PEO}$ content in SPEO was determined from the integral intensity ratio of the signals at $7.1-8.3 \mathrm{ppm}$ (aromatic proton) over the signals at $3.4-3.6 \mathrm{ppm}$ $\left(-\mathrm{OCH}_{2} \mathrm{CH}_{2}-\right)$ in ${ }^{1} \mathrm{HNMR}$ spectra. ${ }^{1} \mathrm{HNMR}$ $\left(\right.$ DMSO- $\left.d_{6}\right), \delta$ (ppm): 7.1-8.3 $(6 \mathrm{H}, \mathrm{ArH}), 3.4-3.6$ (4H, PEO). IR $\left(\mathrm{KBr}, \mathrm{cm}^{-1}\right): 2871,1782,1720$, $1509,1343,1107,723$. Elementary analysis for starlike polymer (SPEO-I-1 in Table I), Calcd: C, 55.45; H, 7.56; N, 1.07. Found: C, 55.46; H, 6.76; $\mathrm{N}, 1.75$.

Amine-terminated Star-like PEO. To a $100 \mathrm{~mL}$ thoroughly dried four-neck flask were charged $0.75 \mathrm{~g}$ $(0.38 \mathrm{mmol})$ of BAPEO (PEO- $\left.1, M_{\mathrm{n}}=2840\right)$ and $7.5 \mathrm{~mL}$ of DMAc under nitrogen flow. Then $0.31 \mathrm{~g}$ of hyperbranched PI (anhydride: $0.3 \mathrm{mmol}$ ) (Core II: 6FDA/TAPA: 2.07:1) in $6 \mathrm{~mL}$ of DMAc was added dropwise through a syringe over $3 \mathrm{~h}$. The reaction mixture was further stirred at room temperature for $3 \mathrm{~h}$. Then $10 \mathrm{~mL}$ of $m$-xylene was added, followed by thermal imidization at $433 \mathrm{~K}$ for $5 \mathrm{~h}$. After cooling to room temperature, the mixture was poured into diethyl ether $\left(\mathrm{Et}_{2} \mathrm{O}\right)$, and the precipitate was washed with $\mathrm{Et}_{2} \mathrm{O}$ and dried in vacuo. The crude product was purified by extracting the unreacted BAPEO with the mixture of $\mathrm{Et}_{2} \mathrm{O} / \mathrm{MeOH}$ (volume ratio = 5:1) and dried in vacuo at room temperature. The PEO content was determined by the method mentioned above. ${ }^{1} \mathrm{HNMR}$ (DMSO- $\left.d_{6}\right), \delta(\mathrm{ppm}): 6.5-8.5(6 \mathrm{H}, \mathrm{ArH})$, 3.4-3.6 (4H, PEO). IR (KBr, cm $\left.{ }^{-1}\right)$ : 2871, 1784, 1720, 1510, 1349, 1105, 725. Anal. calcd for SPEO (PEO content: 56\%): C, 55.95; H, 6.43; N, 2.77. Found: C, 55.59; H, 7.37; N, 2.26 .

Acetamido-terminated Star-like PEO. To the solution of polyamic precursor, which was prepared by the same procedures as described for the amine-terminated SPEO, a mixture of $1.0 \mathrm{~g}$ of triethylamine and $3.0 \mathrm{~g}$ of acetic anhydride was added at room temperature and the reaction mixture was stirred for $3 \mathrm{~h}$ and then at $333 \mathrm{~K}$ for $10 \mathrm{~h}$. The mixture was poured into $\mathrm{Et}_{2} \mathrm{O}$ and the precipitate was washed with $\mathrm{Et}_{2} \mathrm{O}$ and dried in vacuo. The crude product was purified by extracting the unreacted BAPEO with the mixture of $\mathrm{Et}_{2} \mathrm{O}$ / $\mathrm{MeOH}$ (volume ratio $=5: 1$ ) and dried in vacuo at room temperature.

\section{Membrane Preparation}

Membranes were prepared by solution casting method. In the case of methoxy-terminated SPEOs, membranes were prepared by casting their $10 \mathrm{wt} \%$ DMAc solutions onto Teflon dishes, followed by drying in oven and vacuo at $353 \mathrm{~K}$ for $10 \mathrm{~h}$, respectively. Amine-terminated SPEOs were dissolved in methanol to get clear solutions, ethylene glycol diglycidyl ether (EGDE) in methanol was added to the SPEO solutions (the molar ratio between amine group in SPEO and epoxy group in EGDE was controlled to be 2.5:1). The solutions were directly cast onto porous hydrophilic substrates (Millipore: porosity, 70\%; pore size, $0.22 \mu \mathrm{m}$; thickness, $113 \mu \mathrm{m}$ ) to give undoped SPEO 
Table I. Molecular Weight and Physical Properties of The Core Materials, PEO Oligomers, and Star-like PEOs

\begin{tabular}{|c|c|c|c|c|c|c|c|c|c|c|c|c|}
\hline \multirow{2}{*}{ Polymer ${ }^{\mathrm{a}}$} & \multirow{2}{*}{$\begin{array}{l}\text { PEO } \\
(\mathrm{wt} \%)\end{array}$} & \multirow{2}{*}{$\begin{array}{c}\text { Reaction } \\
\text { degree }(\%)\end{array}$} & \multirow{2}{*}{$M_{\mathrm{n}}^{\mathrm{b}}$} & \multirow{2}{*}{$M_{\mathrm{w}} / M_{\mathrm{n}}$} & \multirow{2}{*}{$\begin{array}{c}\text { Viscosity }^{\mathrm{c}} \\
(\mathrm{dL} / \mathrm{g})\end{array}$} & \multirow{2}{*}{$\begin{array}{l}T_{\mathrm{g}} \\
(\mathrm{K})\end{array}$} & \multirow{2}{*}{$\begin{array}{l}T_{\mathrm{m}} \\
(\mathrm{K})\end{array}$} & \multicolumn{5}{|c|}{ Solubility $^{\mathrm{d}}$} \\
\hline & & & & & & & & DMAc & THF & $\mathrm{MeOH}$ & $\mathrm{CH}_{2} \mathrm{Cl}_{2}$ & Tol \\
\hline Core I & & & 1060 & 1.6 & 0.37 & 569 & & + & + & - & - & - \\
\hline Core II & & & 1420 & 2.2 & 0.70 & 585 & & + & + & - & - & - \\
\hline Core III & & & 8400 & 18 & 1.62 & 593 & & + & - & - & - & - \\
\hline PEGME (PEO1) & & & 5000 & - & 0.17 & 209 & 323 & + & + & + & + & + \\
\hline PEGME (PEO2) & & & 2000 & - & 0.06 & 209 & 315 & + & + & + & + & + \\
\hline BAPEO (PEO3) & & & 2840 & 2.2 & 0.07 & 207 & 316 & + & + & + & + & + \\
\hline SPEO-I-1-ME & 78 & 59 & $\mathrm{NM}$ & NM & 0.66 & 221 & 296 & + & - & - & - & - \\
\hline SPEO-II-2-ME & 47 & 45 & 11000 & 7.5 & 0.77 & 233 & ND & + & - & - & - & - \\
\hline SPEO-III-1-ME & 58 & 44 & 20000 & 24 & 1.12 & 223 & ND & + & - & - & - & - \\
\hline SPEO-II-3-AM-1 & 56 & 64 & 4600 & 7.8 & 0.50 & 222 & 298 & + & + & + & + & + \\
\hline SPEO-II-3-AM-2 & 60 & 69 & 4700 & 10.5 & 0.49 & 222 & 298 & + & + & + & + & + \\
\hline SPEO-II-3-AC & 55 & 64 & NM & NM & 0.65 & 231 & 299 & + & +- & +- & +- & +- \\
\hline
\end{tabular}

${ }^{a}$ Molar ratio of 6FDA to TAPA in core: 2.25/1(core I), 2.07/1(core II), 2.0/1(core III); In abbriviation of the star-like PEOs, e.g. SPEO-I-1-ME, "I, II, III" refer to core number, "1, 2, 3" refer to PEO number, -ME refers to methoxy-terminated SPEO, -AM refers to amine-terminated SPEO, -AC refers to acetamido-terminated SPEO. ${ }^{\mathrm{b}} \mathrm{GPC}$ by NMP or THF. ${ }^{\mathrm{c}} 308 \mathrm{~K}$ in DMAc in $0.5 \mathrm{wt} \%$. ${ }^{\mathrm{d}} \mathrm{Key}:+$ : soluble; +-: partially soluble; -: insoluble; NM: not measured; ND: not detectable.

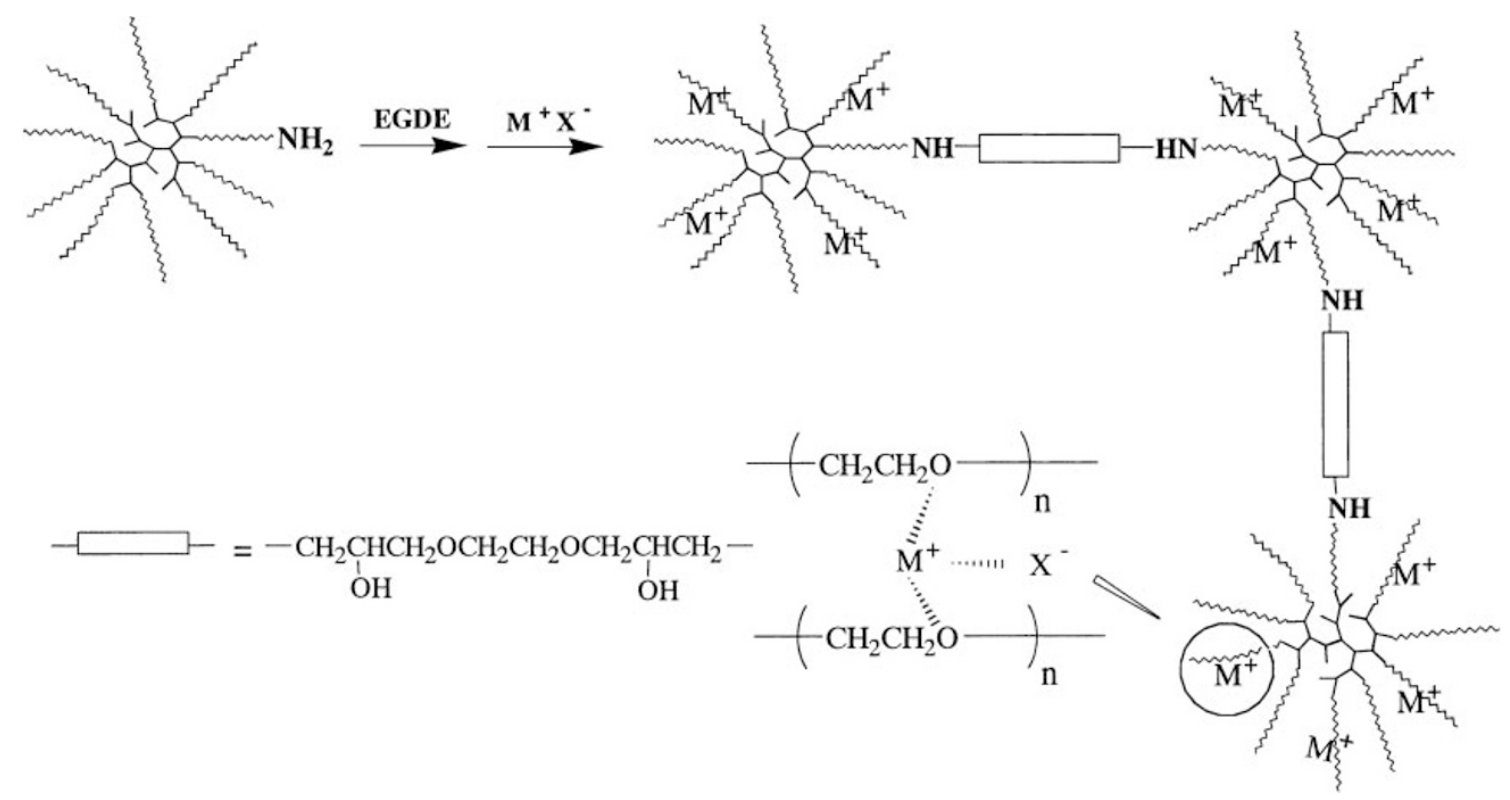

Figure 3. Schematic diagram of preparation of metal ion-doped star-like PEO.

composite membranes. To obtain metal ion-doped composite membranes, the methanol solutions of an amine-terminated SPEO mixed with metal salts such as $\mathrm{AgBF}_{4}, \mathrm{KF}, \mathrm{LiClO}_{4}$ and $\left(\mathrm{CH}_{3}\right)_{4} \mathrm{NF}$ were cast onto the porous hydrophilic substrates (see Figure 3). The concentration of the SPEO solution was controlled to be $15-20 \mathrm{wt} \%$. The membranes were first maintained at room temperature for $2 \mathrm{~h}$ and then $333 \mathrm{~K}$ for $10 \mathrm{~h}$. Then the membranes were dried in vacuo at $333 \mathrm{~K}$ for $5 \mathrm{~h}$.

\section{Measurements}

Infrared (IR) spectra were recorded on a Horiba FT200 spectrometer as $\mathrm{KBr}$ pellets. ${ }^{1} \mathrm{H}$ NMR spectra were recorded on a JEOL EX270 $(270 \mathrm{MHz})$ instru- ment. Inherent viscosity was measured in DMAc solution at a concentration of $0.5 \mathrm{wt} \%$ at $308 \mathrm{~K}$ with an Ubbelodhe viscometer. Differential scanning calorimetry (DSC) was performed with a DSC-5200 by SEI$\mathrm{KO}$ Co. at a heating rate of $5 \mathrm{~K} / \mathrm{min}$. Glass transition temperature $\left(T_{\mathrm{g}}\right)$ and melting temperature $\left(T_{\mathrm{m}}\right)$ were determined from the onset points of the signals on the first heating run. Dynamic mechanical spectroscopy was performed with a mechanical spectrometer (itk DVA-200) at $10 \mathrm{~Hz}$ and a peak strain amplitude of $0.15 \%$. Gel permeation chromatography (GPC) was performed with HLC-8020 apparatus (Column: Shodex KD-80M) and LC-10AS apparatus (Column: HSG-10), respectively. NMP and THF were used as eluants at a flow rate of $1.0 \mathrm{~mL} / \mathrm{min}$, respectively. 
Polymer solutions containing $0.05 \mathrm{M}$ of $\mathrm{LiCl}$ and $0.05 \mathrm{M}$ of phosphoric acid were filtered through a $0.5 \mu \mathrm{m}$ PTFE filter prior to injecting into the column. Molecular weights were calculated against PEO or polystyrene standards, respectively.

Permeability coefficient $P$ (in Barrer, 1 Barrer = $1 \times 10^{-10} \mathrm{~cm}^{3}$ (STP) $\mathrm{cm}^{-1} \mathrm{~s}^{-1} \mathrm{~cm} \mathrm{Hg}^{-1}$ ) was measured by means of a vacuum time-lag method. The ideal selectivity is calculated from the permeability ratio of the gas pair as shown in Eq 1:

$$
\alpha_{\mathrm{i}, A / B}=P_{A} / P_{B}
$$

where $P_{A}$ and $P_{B}$ refer to the permeability coefficients of pure gases $A$ and $B$, respectively. The diffusion coefficient $D$ is calculated according to Eq 2:

$$
D=L^{2} / 6 \theta
$$

where $L$ is the thickness of the membrane and $\theta$ is the time-lag. Apparent solubility coefficient, $S$, is calculated from $S=P / D$.

In the case of mixed gases, separation factor $\alpha$ defined by Eq 3 is used instead of the ideal selectivity.

$$
\alpha=[y(1-x)] /[x(1-y)]
$$

where $x$ and $y$ are molar fraction of gases in feed and permeate determined by gas chromatography, respectively.

\section{RESULTS AND DISCUSSION}

\section{Synthesis and Characterization of Core and Star-like PEO}

We have previously reported the synthesis of two kinds of hyperbranched PIs from 6FDA and TAPA by controlling the addition order and the molar ratio of monomers. ${ }^{6}$ The schematic diagram of the synthesis of anhydride-terminated hyperbranched PIs is shown in Figure 1. Three types of cores with different molecular sizes were prepared by controlling the molar ratios of 6FDA over TAPA. The SPEOs were synthesized from these cores and PEO arms with the different block lengths and terminal groups (as shown in Figure 2). The mole of PEO arm was controlled to be excess over the mole of anhydride in core. The PEO content in the corresponding SPEO was determined from the ${ }^{1} \mathrm{HNMR}$ spectrum. The reaction degree was calculated from the ratio of actual and ideal PEO contents in the SPEO. The typical results are listed in Table I. The PEO content and the reaction degree depended on the size of core. The reaction degree was generally low for the methoxy-terminated SPEOs and slightly higher for the amine-terminated ones. An increase in the feed ratio of PEGME or BAPEO with a prolonged reaction time hardly enhanced the reaction degree. The larger core gave the lower reaction de- gree, suggesting the anhydride groups in inner side of the larger core were less reactive probably because of steric hindrance.

The physical properties of various cores and SPEOs are also summarized in Table I. The SPEOs were readily soluble in aprotic solvents such as DMAC. The amine-terminated SPEOs were soluble even in toluene and methanol, indicating excellent solubility. This suggests that the crosslinking reaction of BAPEO between PI cores was substantially negligible. The acetamido-terminated SPEO (SPEO-II-3-AC) did not show such good solubility, although it had the same structure as the amine-terminated SPEO (SPEO-II-3AM) except for the terminal groups. The methoxy-terminated SPEOs (SPE-I-ME, -II-2-ME, -III-1-ME) were not soluble in THF, methanol and toluene, in which PEOs were soluble. These indicate the terminal groups have a large effect on solubility of SPEOs.

The SPEOs showed only the lower glass transition temperature $\left(T_{\mathrm{g}}\right)$ corresponding to the PEO arms, but not the higher one corresponding to the hyperbranched PI cores, whereas the PEO-PIs showed both $T_{\mathrm{g}} \mathrm{s}$. Furthermore, the thermo-mechanical property of the SPEOs was different from that of the linear PEO-PIs. As shown in Figure 4, the dynamic modulus $E^{\prime}$ of SPEO-II-3-AM (PEO: $56 \mathrm{wt} \%$ ) decreased drastically down to $1 \times 10^{7} \mathrm{~Pa}$ around $T_{\mathrm{m}}$ of the PEO segment $(298 \mathrm{~K})$, whereas the $E^{\prime}$ of the linear PEO-PI (PEO: $52 \mathrm{wt} \%$ ) was maintained at a much larger level of $3 \times 10^{8} \mathrm{~Pa}$ at $323 \mathrm{~K}$ and decreased down to $1 \times$ $10^{7} \mathrm{~Pa}$ around $T_{\mathrm{g}}$ for the PI segment $(521 \mathrm{~K}){ }^{8}$ These results indicate a clear difference in the morphology

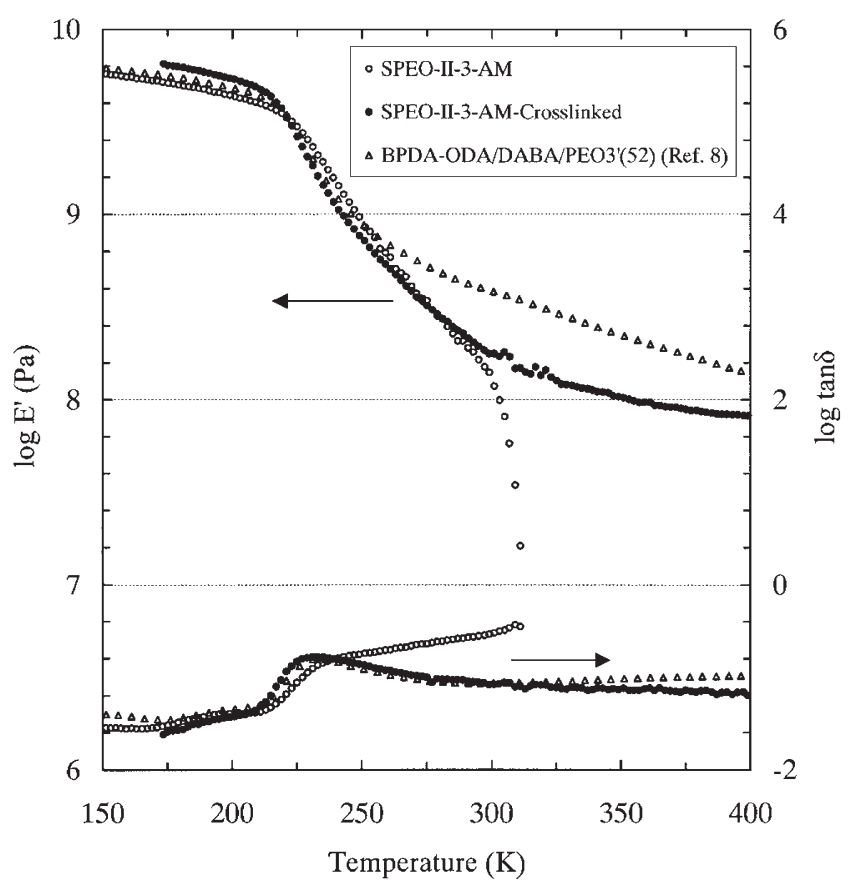

Figure 4. Dynamic mechanical spectroscopy of star-like PEO polymers. 
between the SPEOs and PEO-PIs. In the case of SPEOs, the PI cores are isolated in the PEO arm matrix and can not aggregate each other to form the PI domains. On the other hand, PEO-PIs have the microphase-separated structure composed of PEO domains and PI domains. ${ }^{8}$

The $T_{\mathrm{g}} \mathrm{S}$ of the SPEOs were higher by $15-20 \mathrm{~K}$ than those of the corresponding PEO arms. They were a little higher than the $T_{\mathrm{g}} \mathrm{s}$ of the linear PEO-PIs with similar PEO block length ${ }^{8}$ and similar to those of the PEO-segmented block copolyamides (PEO-PAs), ${ }^{9}$ indicating a difference in the segmental mobility of PEO between SPEOs and PEO-PIs. In the case of PEO-segmented block copolymers, the increase in $T_{\mathrm{g}}$ of PEO component depended on the hard segment content in the PEO domain, a measure of the incompleteness of microphase separation, and was in the order of polyurethane $>$ polyamide $\gg$ polyimide. ${ }^{9}$ In the case of SPEOs, the PI cores are isolated in the PEO arm matrix and can not aggregate each other to form PI domains. Therefore, the SPEOs are likely to have a larger content of boundary layers, or intermediate phases containing PI and PEO segments, than the PEO-PIs. Another example that polymer physical properties were significantly affected by the intermediate phases was reported in literature. ${ }^{10}$

The thermo-mechanical property of SPEOs was significantly improved by cross-linking treatment. EGDE, a difunctional compound having two epoxy groups per molecule, was used to react with amine- terminated SPEOs (see Figure 3). After the cross-linking, the films were insoluble in any solvents and maintained a much higher $E^{\prime}$ value of $1 \times 10^{8} \mathrm{~Pa}$ at $373 \mathrm{~K}$.

For gas permeation experiments, composite membranes of amine-terminated SPEO on porous hydrophilic substrate were prepared and the cross-linking reaction was conducted during the membrane casting process. For metal ion-doped SPEOs, the metal salts and EGDE were added into polymer solutions at the same time and then cast into membranes (see Figure 3).

\section{Gas Permeation Properties of Star-like PEO Mem- branes}

$\mathrm{CO}_{2} / \mathrm{N}_{2}$ and $\mathrm{CO}_{2} / \mathrm{H}_{2}$ Permeation Properties The permeability coefficient $P$ and the ideal selectivity $\alpha_{\mathrm{i}}$ of $\mathrm{CO}_{2} / \mathrm{N}_{2}$ and $\mathrm{CO}_{2} / \mathrm{H}_{2}$ for SPEO membranes are listed in Table II together with the data for the related polymers cited from references. ${ }^{7,8,13,15}$ The $P$ and $\alpha_{\mathrm{i}}$ were independent of feed pressure up to $5 \mathrm{~atm}$, as in the case of PEO-segmented block copolymers. ${ }^{8,9}$ The SPEO membranes were much more permeable to $\mathrm{CO}_{2}$ than to $\mathrm{N}_{2}$ and even to $\mathrm{H}_{2}$, which is characteristic of PEO-based membranes. This is attributed to both the high solubility of $\mathrm{CO}_{2}$ due to the high affinity of $\mathrm{CO}_{2}$ to polar PEO matrix and the less size-dependent diffusivity of penetrant in rubbery PEO matrix. ${ }^{8}$ However, the SPEOs were a little inferior to the PEO-PIs in the separation performance. The $P_{\mathrm{CO}_{2}}$ values were about two times smaller for the SPEOs than

Table II. $\mathrm{CO}_{2}, \mathrm{~N}_{2}$ and $\mathrm{H}_{2}$ Permeation Properties of Star-like PEO and Other Polymer Membranes at $308 \mathrm{~K}$

\begin{tabular}{|c|c|c|c|c|c|c|c|c|}
\hline \multirow{2}{*}{ Membrane $(\mathrm{PEO} w \mathrm{t} \%)^{\mathrm{a}}$} & \multirow{2}{*}{$\begin{array}{l}\text { Dopant } \\
\text { (wt\%) }\end{array}$} & \multirow{2}{*}{$\begin{array}{c}\text { Pressure } \\
\text { (atm) }\end{array}$} & \multicolumn{3}{|c|}{ Permeability [Barrer] } & \multicolumn{2}{|c|}{ Permeability ratio } & \multirow{2}{*}{ Ref. } \\
\hline & & & $\mathrm{CO}_{2}$ & $\mathrm{~N}_{2}$ & $\mathrm{H}_{2}$ & $\mathrm{CO}_{2} / \mathrm{N}_{2}$ & $\mathrm{CO}_{2} / \mathrm{H}_{2}$ & \\
\hline SPEO-III-1-ME (58) & - & 2 & 55 & 1.1 & 9.1 & 50 & 6.0 & This \\
\hline \multirow[t]{7}{*}{ SPEO-II-3-AM (60) } & - & 5 & 40 & 0.71 & 6.0 & 55 & 6.5 & This \\
\hline & $\operatorname{AgBF}_{4}(30)$ & 2 & 5.8 & 0.12 & - & 49 & - & This \\
\hline & & 5 & 6.1 & 0.12 & - & 50 & - & This \\
\hline & $\left(\mathrm{CH}_{3}\right)_{4} \mathrm{NF}(15)$ & 2 & 52 & 1.2 & 10.6 & 45 & 4.9 & This \\
\hline & $\left(\mathrm{CH}_{3}\right)_{4} \mathrm{NF}(23)$ & 2 & 35 & 2.5 & 15 & 14 & 2.4 & This \\
\hline & $\mathrm{KF}(15)$ & 2 & 36 & 0.89 & 8.1 & 41 & 4.4 & This \\
\hline & $\mathrm{LiClO}_{4}(15)$ & 5 & 5.0 & 0.13 & 1.6 & 47 & 3.2 & This \\
\hline \multicolumn{9}{|l|}{ PEO-PI } \\
\hline BPDA-ODA/DABA/PEO3' (58) & - & 2 & 93 & 1.84 & - & 50 & - & 8 \\
\hline 6FDA-ODA/PEO3' (51) & - & 2 & 26 & 0.52 & - & 50 & - & 8 \\
\hline PMDA/mPD/PEO3' (56) & - & 1 & 99 & 2.0 & 10.8 & 50 & 9.2 & 8 \\
\hline \multicolumn{9}{|l|}{ Branched polyether } \\
\hline P(EO/EM/AGE) (85) & - & 1 & 770 & 16.7 & 78 & 46 & 9.9 & 13 \\
\hline \multicolumn{9}{|l|}{ Hyperbranched PI } \\
\hline 6FDA-TAPA & - & 1 & 65 & 2.2 & 86 & 30 & 0.76 & 7 \\
\hline $\mathrm{PEO}^{\mathrm{b}}$ & - & 6.8 & 12 & - & 1.3 & - & 9.3 & 15 \\
\hline $\mathrm{PEO}^{\mathrm{b}}$ & $\left(\mathrm{CH}_{3}\right)_{4} \mathrm{NF}(22)$ & 6.8 & 19 & - & 1.46 & - & 13 & 15 \\
\hline
\end{tabular}

aBPDA: 3,3',4,4'-biphenyltetracarboxylic dianhydride, ODA: 4,4'-diaminodiphenyl ether, DABA: 3,5-diaminobenzoic acid, PMDA: pyromellitic dianhydride, mPD: 1,3-phenylenediamine, PEO3': BAPEO $(n=52)$, EO: ethylene oxide, EM: 2-(2-methoxyethoxy)ethyl glycidyl ether, AGE: allylglycidyl ether. ${ }^{b}$ Measured at $298 \mathrm{~K}$. 
for the PEO-PIs with the similar PEO content, and the $\alpha_{\mathrm{i}}$ values of $\mathrm{CO}_{2} / \mathrm{H}_{2}$ were $30 \%$ smaller for the former, although those of $\mathrm{CO}_{2} / \mathrm{N}_{2}$ were similar between them. This is attributed probably to some difference in the morphology. In the case of SPEOs, the PI cores are isolated in the PEO arm matrix and can not aggregate together to form PI domains. Therefore, the SPEOs are likely to have a larger content of intermediate phases containing PI and PEO segments than the PEO-PIs, resulting in the lower $P_{\mathrm{CO}_{2}}$ and the lower selectivity.

Recently, self-standing and crosslinked elastomer membranes were prepared from PEO-based branched polyethers $(\mathrm{P}(\mathrm{EO} / \mathrm{EM} / \mathrm{AGE}))$ which were in amorphous state in spite of high molecular weight. They displayed extremely high $P_{\mathrm{CO}_{2}}$ values ranging from

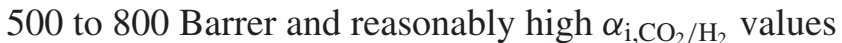
of about 10 at $308 \mathrm{~K}^{13}$ On the other hand, a PEO membrane with a high molecular weight displayed very low $P_{\mathrm{CO}_{2}}$ values ranging from 12 to 17 Barrer and reasonably high $\alpha_{\mathrm{i}, \mathrm{CO}_{2} / \mathrm{H}_{2}}$ values ranging from 7.4 to 9.9 depending on the feed pressure at $308 \mathrm{~K}$, probably because of high degree of crystallinity. ${ }^{15}$ Interestingly, it was found that the addition of $22 \mathrm{wt} \%$ of $\left(\mathrm{CH}_{3}\right)_{4} \mathrm{NF}$ into the PEO membrane enhanced both $P_{\mathrm{CO}_{2}}$ and $\alpha_{\mathrm{i}, \mathrm{CO}_{2} / \mathrm{H}_{2}}$, as listed in Table II. ${ }^{15}$ Therefore, in this study, the effect of dopants on $P_{\mathrm{CO}_{2}}$ and $\alpha_{\mathrm{i}, \mathrm{CO}_{2} / \mathrm{H}_{2}}$ for the SPEO membranes was also investigated. The results are listed in Table II. The doping of $15 \mathrm{wt} \%$ (corresponding to $25 \mathrm{wt} \%$ in PEO matrix) of $\left(\mathrm{CH}_{3}\right)_{4} \mathrm{NF}$ increased $P_{\mathrm{CO}_{2}}$ by $30 \%$, but decreased $\alpha_{\mathrm{i}, \mathrm{CO}_{2} / \mathrm{H}_{2}}$ by $25 \%$. The further doping of $\left(\mathrm{CH}_{3}\right)_{4} \mathrm{NF}$ decreased both $P_{\mathrm{CO}_{2}}$ and $\alpha_{\mathrm{i}, \mathrm{CO}_{2} / \mathrm{H}_{2}}$. Other dopants such as $\mathrm{LiClO}_{4}$ also decreased both $P_{\mathrm{CO}_{2}}$ and $\alpha_{\mathrm{i}, \mathrm{CO}_{2} / \mathrm{H}_{2}}$.

\section{$\mathrm{C}_{2} \mathrm{H}_{4} / \mathrm{C}_{2} \mathrm{H}_{6}$ Permeation Properties}

As for ethylene and ethane, the time-lag $\theta$ was large enough to be measured accurately. Apparent diffusion and solubility coefficients, $D$ and $S$ were evaluated. Table III lists the $P, D, S$ values and their ratios of $\mathrm{C}_{2} \mathrm{H}_{4} / \mathrm{C}_{2} \mathrm{H}_{6}$ for a SPEO membrane and $\mathrm{AgBF}_{4}$-doped SPEO membranes, together with the $P$ data for the related doped membranes. ${ }^{14,16}$ For the SPEO membrane, the $P$ was independent of feed pressure. On the other hand, for the $\mathrm{AgBF}_{4}$-doped SPEO membranes, the $P_{\mathrm{C}_{2} \mathrm{H}_{4}}$ increased by $70-80 \%$ with increasing the feed pressure up to $10 \mathrm{~atm}$, whereas the $P_{\mathrm{C}_{2} \mathrm{H}_{6}}$ hardly

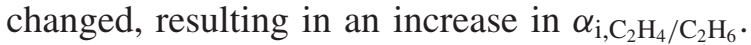

The undoped SPEO membrane showed low separation performance for $\mathrm{C}_{2} \mathrm{H}_{4} / \mathrm{C}_{2} \mathrm{H}_{6}$, namely, a relatively

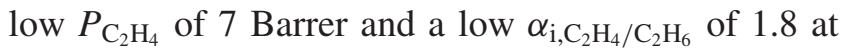
$308 \mathrm{~K}$. The doping of $\mathrm{AgBF}_{4}$ led to a decrease in

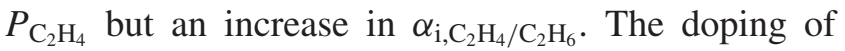
$30 \mathrm{wt} \%$ (corresponding to $50 \mathrm{wt} \%$ in PEO matrix) of $\mathrm{AgBF}_{4}$ caused a significant decrease in $P_{\mathrm{C}_{2} \mathrm{H}_{4}}$ by a fac-

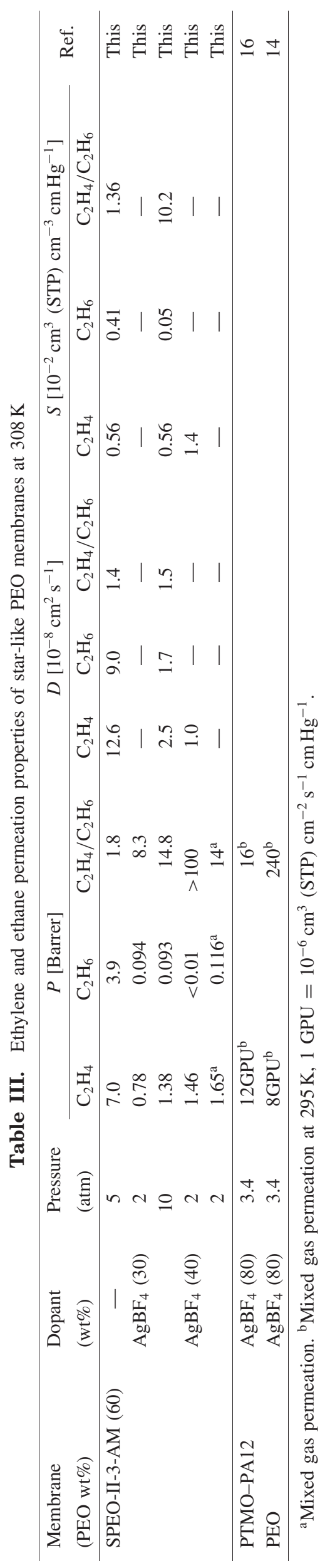


tor of $80 \%$. This was attributed only to a decrease in $D_{\mathrm{C}_{2} \mathrm{H}_{4}}$ and $S_{\mathrm{C}_{2} \mathrm{H}_{4}}$ hardly changed. On the other hand, $P_{\mathrm{C}_{2} \mathrm{H}_{6}}$ decreased by a much larger factor of $98 \%$ due to both significant decreases in $D_{\mathrm{C}_{2} \mathrm{H}_{6}}$ and $S_{\mathrm{C}_{2} \mathrm{H}_{6}}$. The doping with silver salt decreased $S_{\mathrm{C}_{2} \mathrm{H}_{6}}$ by a factor of 8 and increased the solubility selectivity by the same

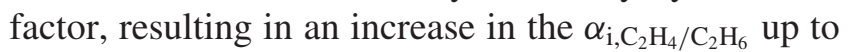
15. Raising the $\mathrm{AgBF}_{4}$ loading from $30 \mathrm{wt} \%$ to $40 \mathrm{wt} \%$ (corresponding to $67 \mathrm{wt} \%$ in PEO matrix) slightly increased $P_{\mathrm{C}_{2} \mathrm{H}_{4}}$, but significantly decreased $P_{\mathrm{C}_{2} \mathrm{H}_{6}}$ below the measurement limit. The slight increase in $P_{\mathrm{C}_{2} \mathrm{H}_{4}}$ was due to a large increase in $S_{\mathrm{C}_{2} \mathrm{H}_{4}}$, which more than compensated for a large decrease in $D_{\mathrm{C}_{2} \mathrm{H}_{4}}$. The $\pi$-bonding interaction between $\mathrm{Ag}^{+}$ and ethylene molecule enhances the ethylene solubility. The silver salt dissolves into the PEO matrix due to the interaction between $\mathrm{Ag}^{+}$and ether oxygen. It is reasonably considered that the doping effect resulted in denser packing of PEO matrix and significantly reduced segmental mobility of PEO. This is the reason for the large decreases in $S_{\mathrm{C}_{2} \mathrm{H}_{6}}$ as well as for the large decreases in $D$. The significant reduction in the ethane solubility with the doping of $\mathrm{AgBF}_{4}$ is a kind of the "salting-out effect" of the silver salt, and has been also observed for $\mathrm{AgBF}_{4}$-doped PTMO-PA12 membranes. ${ }^{17}$ With the loading of $50 \mathrm{wt} \%$, pinhole-free membranes could not be fabricated because of the deposition of the dopant. This means that the PEO matrix of the SPEOs is inferior to PEO homopolymer and PTMO-PA12 in dissolving capacity to $\mathrm{AgBF}_{4}$.

For the SPEO membrane doped with $40 \mathrm{wt} \%$ $\mathrm{AgBF}_{4}$, the mixed gas permeation experiments were carried out using a mixed gas of $\mathrm{C}_{2} \mathrm{H}_{4} / \mathrm{C}_{2} \mathrm{H}_{6}(\mathrm{v} / \mathrm{v}$ 50/50). The results are listed in Table III. The $P_{\mathrm{C}_{2} \mathrm{H}_{4}}$ was 1.65 Barrer and the selectivity of $\mathrm{C}_{2} \mathrm{H}_{4} / \mathrm{C}_{2} \mathrm{H}_{6}$ was 14 at $2 \mathrm{~atm}$ and $308 \mathrm{~K}$. Compared with the single component permeation, the mixed gas permeation gave the much lower permselectivity. This is because the ethane permeability significantly increased with the coexisting ethylene. As mentioned above, the enhancement in the ideal selectivity of $\mathrm{C}_{2} \mathrm{H}_{4} / \mathrm{C}_{2} \mathrm{H}_{6}$ was due mainly to the drastic reduction in the ethane solubility. It is likely that "the salting-out effect" of $\mathrm{AgBF}_{4}$ is weakened with the bonding of ethylene molecules with $\mathrm{Ag}^{+}$ions. The much lower permselectivity of olefin over paraffin in the mixed gas permeation compared with the ideal selectivity has been commonly observed for this type of solid polymer electrolyte membrane. ${ }^{14,19}$

\section{CONCLUSIONS}

1. Star-like polymers were synthesized from anhydride-terminated hyperbranched PI cores and PEO arms. The amine-terminated SPEOs dis- played better solubility property than the methoxy- and acetamido-terminated ones. The terminal groups had a large effect on solubility of the SPEOs.

2. The star-like PEOs showed only the lower $T_{\mathrm{g}} \mathrm{s}$ of which the values were higher by $15-20 \mathrm{~K}$ than those of the corresponding PEO arms and showed a drastic decrease in the $E^{\prime}$ around $T_{\mathrm{m}}$ of the PEO arms $(298 \mathrm{~K})$, suggesting the morphology that each PI core is isolated in the PEO arm matrix with a larger content of intermediate phases.

3. The thermo-mechanical property of amine-terminated SPEOs was significantly improved by cross-linking with EGDE.

4. The star-like PEO membranes were much more permeable to $\mathrm{CO}_{2}$ than to $\mathrm{N}_{2}$ and even to $\mathrm{H}_{2}$, which is characteristic of PEO-based membranes. However, they were slightly inferior to the PEO-PIs in the separation performance, probably due to the difference in the morphology.

5. The $\mathrm{AgBF}_{4}$-doped star-like $\mathrm{PEO}$ membrane with a loading of $40 \mathrm{wt} \%$ (corresponding to $67 \mathrm{wt} \%$ in PEO matrix) showed a very high ideal selectivity of $\mathrm{C}_{2} \mathrm{H}_{4} / \mathrm{C}_{2} \mathrm{H}_{6}$ of more than 100 with a low $P_{\mathrm{C}_{2} \mathrm{H}_{4}}$ of 1.5 Barrer in single-component permeation at $2 \mathrm{~atm}$ and $308 \mathrm{~K}$, which was due mainly to the drastic reduction in the ethane solubility with the doping, or a kind of the "salting-out effect" of the silver salt. However, the permselectivity decreased down to 14 in mixed gas permeation due to a significant increase in $P_{\mathrm{C}_{2} \mathrm{H}_{6}}$, because "the salting-out effect" of $\mathrm{AgBF}_{4}$ was likely weakened with the bonding of ethylene molecules with $\mathrm{Ag}^{+}$ions.

\section{REFERENCES}

1. W. J. Koros and G. K. Fleming, J. Membrane Sci., 83, 1 (1993).

2. S. A. Stern, J. Membrane Sci., 94, 1 (1994).

3. K. Tanaka, O. Osada, H. Kita, and K. Okamoto, J. Polym. Sci., Part B: Polym. Phys., 33, 1907 (1995).

4. K. Tanaka, A. Taguchi, J. Hao, H. Kita, and K. Okamoto, J. Membrane Sci., 121, 197 (1996).

5. R. L. Burns and W. J. Koros, J. Membrane Sci., 211, 299 (2003).

6. J. Fang, H. Kita, and K. Okamoto, Macromolecules, 33, 4639 (2000).

7. J. Fang, H. Kita, and K. Okamoto, J. Membrane Sci., 182, 245 (2001).

8. K. Okamoto, M. Fujii, S. Okamyo, H. Suzuki, K. Tanaka, and H. Kita, Macromolecules, 28, 6950 (1995).

9. M. Yoshino, K. Ito, H. Kita, and K. Okamoto, J. Polym. Sci., Part B: Polym. Phys., 38, 1707 (2000).

10. J. Fang, K. Tanaka, H. Kita, K. Okamoto, and Y. Ito, 
J. Polym. Sci., Part B: Polym. Phys., 38, 1123 (2000).

11. H. Suzuki, K. Tanaka, H. Kita, K. Okamoto, H. Hoshino, T. Yoshinaga, and Y. Kusuki, J. Membrane Sci., 146, 31 (1998).

12. Y. Hirayama, N. Tanihara, Y. Kusuki, Y. Kase, K. Haraya, and K. Okamoto, J. Membrane Sci., 163, 373 (1999).

13. M. Yoshino, H. Kita, K. Okamoto, M. Tabuchi, and T. Sakai, Trans. Mater. Res. Soc. Jpn., 27, 419 (2002).

14. I. Pinnau and L. G. Toy, J. Membrane Sci., 184, 39 (2001).

15. H. Lin, X. Jiang, N. P. Patel, R. J. Spontak, and B. D.
Freeman, Polym. Mater. Sci. Eng., 85, 146 (2001).

16. A. Morisato, Z. He, I. Pinau, and T. Merkel, Polym. Mater. Sci. Eng., 85, 96 (2001).

17. T. C. Merkel, Z. He, A. Morisato, and I. Pinau, Chem. Commun., 13, 1596 (2003).

18. A. Heise, C. Nguyen, R. Malek, J. L. Hedrick, C. W. Frank, and R. D. Miller, Macromolecules, 33, 2346 (2000).

19. S. Hong, J. Y. Kim, and Y. S. Kang, Polym. Adv. Technol., 12, 177 (2001). 